\title{
Heretical Science
}

\author{
Ian L. Jaucian
}

\section{Introduction}

Are there such things as hidden dimensions? What would it mean to us if they did? What is a dimension, anyway?

This exhibition attempts to tackle these questions and scrutinize our common and oftentimes intuitive ideas about space. There is no physical law that can disprove the presence of these hidden dimensions. But for that matter there is also close to nothing that proves they exist at all. However, if hard evidence for such heretical ideas will be found someday, the consequences for mankind's relationship with reality would be on an unimaginable level.

\section{Defining Dimensions}

We can define "dimensions" in so many words and numbers, but for the purpose of our discussion, I think it would be best to work by analogy. Let's start with our concept of a geometric point. It's a small dot that is infinitely tiny. We can call the point a zero-dimensional object because there is zero amount of coordinates you need to locate any object found in it. Since it's infinitely small, the object can only exist in that single little pocket of space.

Now using this logic, let us look at the case of the 1-dimensional line: there is 1 piece of information you can give to locate a point in it.

"The lamp post is 10 feet high."

"The fly is ten meters away from the edge of the string." 


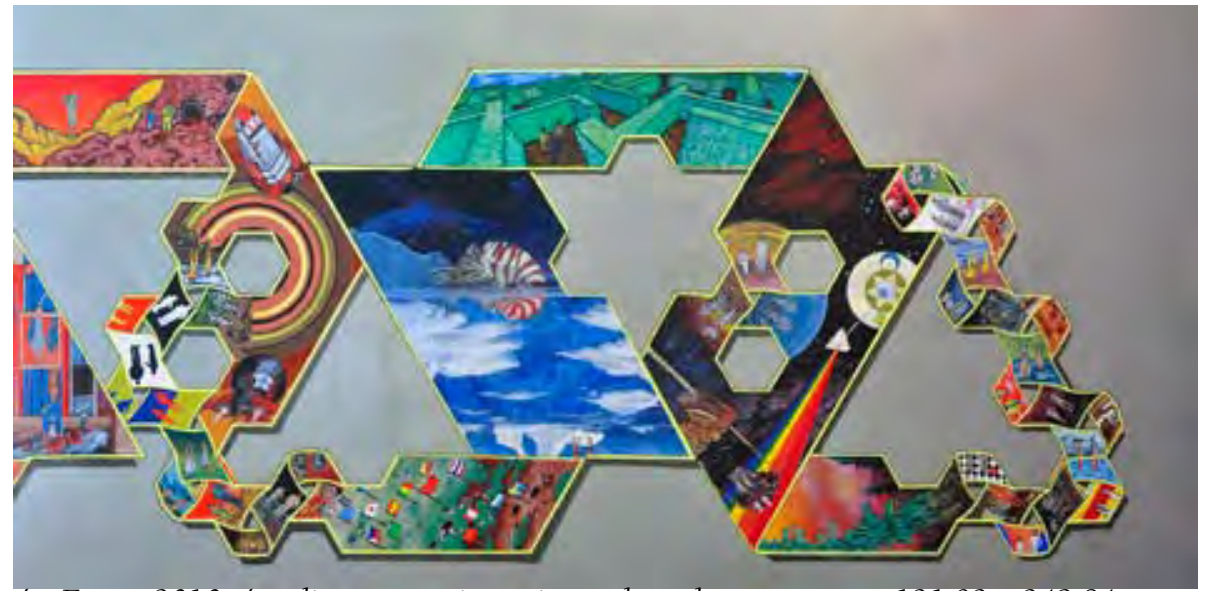

An Event, 2010, Acrylic, automotive paint and marker on canvas, $121.92 \times 243.84 \mathrm{~cm}$

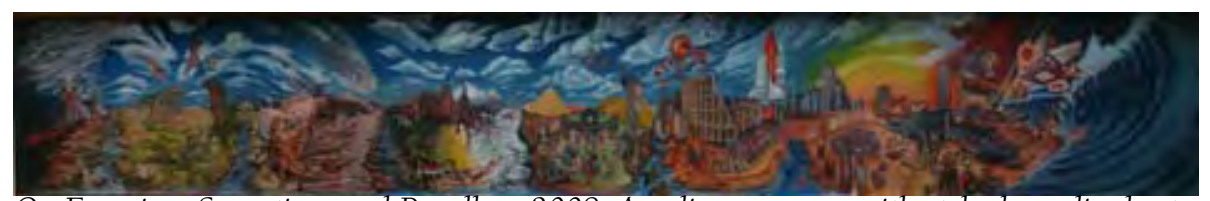

On Focusing, Spacetime and Parallax, 2008, Acrylic on canvas with etched acrylic sheets, $60.96 \times 365.76 \mathrm{~cm}$ (detailed images on page 143)

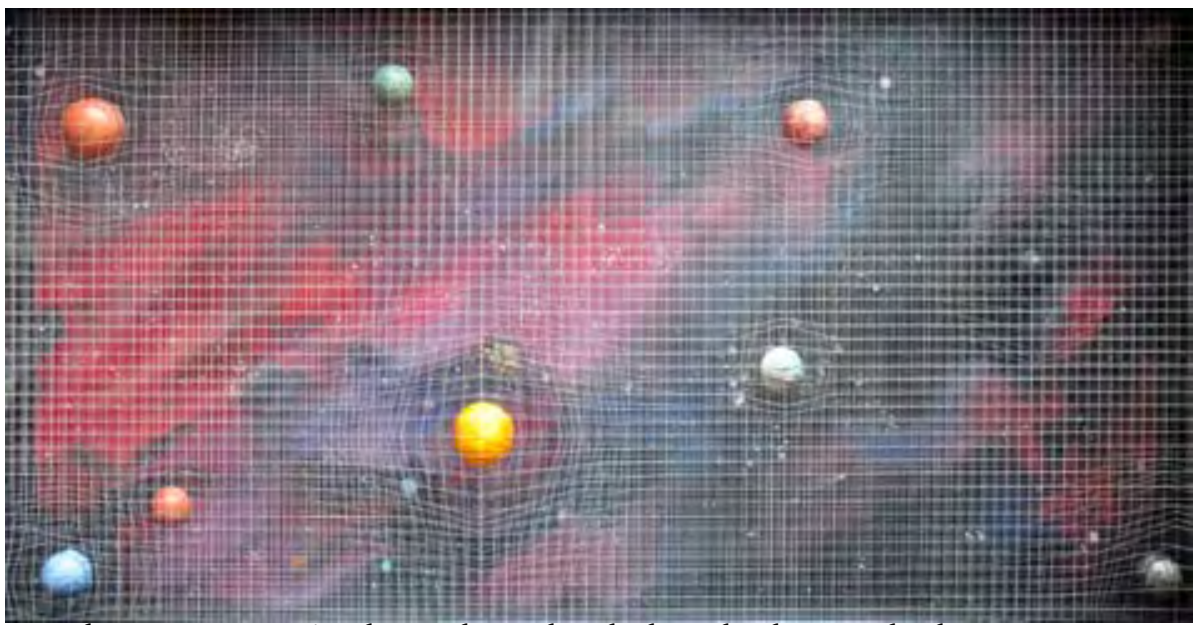

Literal Gravity, 2009, Acrylic on plywood, etched acrylic sheets and polystyrene, $64 \times 125$ $x 18.5 \mathrm{~cm}$ 
In a 2-dimensional plane:

"That piece of paper is 11 inches long and 8.5 inches wide."

"Captain, I've located Skull Island on the map. It's at +40.689060 and -74.044636."

In a 3-dimensional space:

"This box is 6 feet tall, 4 meters long and 12 hectares wide."

"I have a meeting with Mr. Yamamoto in his office which is in the 12th floor of the Hibiki Building located between Malunggay Avenue and Comet Street."

And that's as far as it goes-for now. As beings only capable of perceiving 3 dimensions, we are left with two possibilities. It's either there really are just 3 , or that the hidden ones are simply beyond our perception, whether it be a limitation of biology, consciousness, or the metaphysical. Multiple dimensions can, however, be expressed in mathematical means or by visual analogy.

\section{The Fourth Dimension}

It is worth noting that some people consider "time" as the fourth dimension since it too is a piece of information so to speak:

"I have a 12:25pm meeting with Mr. Yamamoto in his office which is in the 12th floor of the Hibiki Building located between Malunggay Avenue and Comet Street."

But for the purpose of this segment, only spatial dimensions are relevant.

\section{The Physicists' Wager}

Some physicists are betting their whole careers that hidden dimensions exist. Why? 
Apparently, man's understanding of the universe rests upon the shoulders of two distinct fields of physics: general reativity and quantum mechanics. General relativity can simply be thought of as the study of the large, and its complementary, quantum mechanics, the study of the very small. While one describes the orbits of planets, the other tracks the path of atoms. One makes GPS accurate, the other makes it possible to create electronic devices that make GPS readings.

Now here is the predicament. These two bodies of knowledge can never equate in a mathematical arena. This incompatibility has prevented our race from creating a "theory-of-everything," so to speak. Now this is the interesting bit: dimensions can be expressed mathematically. And when you include hidden dimensions in the equations that describe general reativity and quantum mechanics, the two theories suddenly make sense combined! Relatively new ideas such as String Theory or M-Theory are using such bold concepts for the scaffolds of a grand theory that promises supreme understanding of the fabric of reality.

It's just too bad that we lack the technology to prove (or disprove) it.

\section{Our Imperfect Eyes}

We have to come to terms with the fact that vision and perception are two very different things. Optical illusions explored by artists and mathematicians alike underscore this phenomenon. Now since the sense that takes priority in understanding the nature of space is vision, we can clearly assume that we cannot fully trust what we see.

Take, for example, its subtle manifestation in the concept of parallel lines. We can easily conceive the idea. We can draw it, we can describe it in words. Yet we can never truly see parallel lines. The mere fact we have two eyes that have a focal point at all times curves our picture of the world. Even if you cover one eye, the spherical eyeball inevitably curves our lens. But even so, the brain compensates and allows us to understand three dimensions with utmost accuracy. This tells us two things:

1. What one perceives is not necessarily the actual nature of things. 


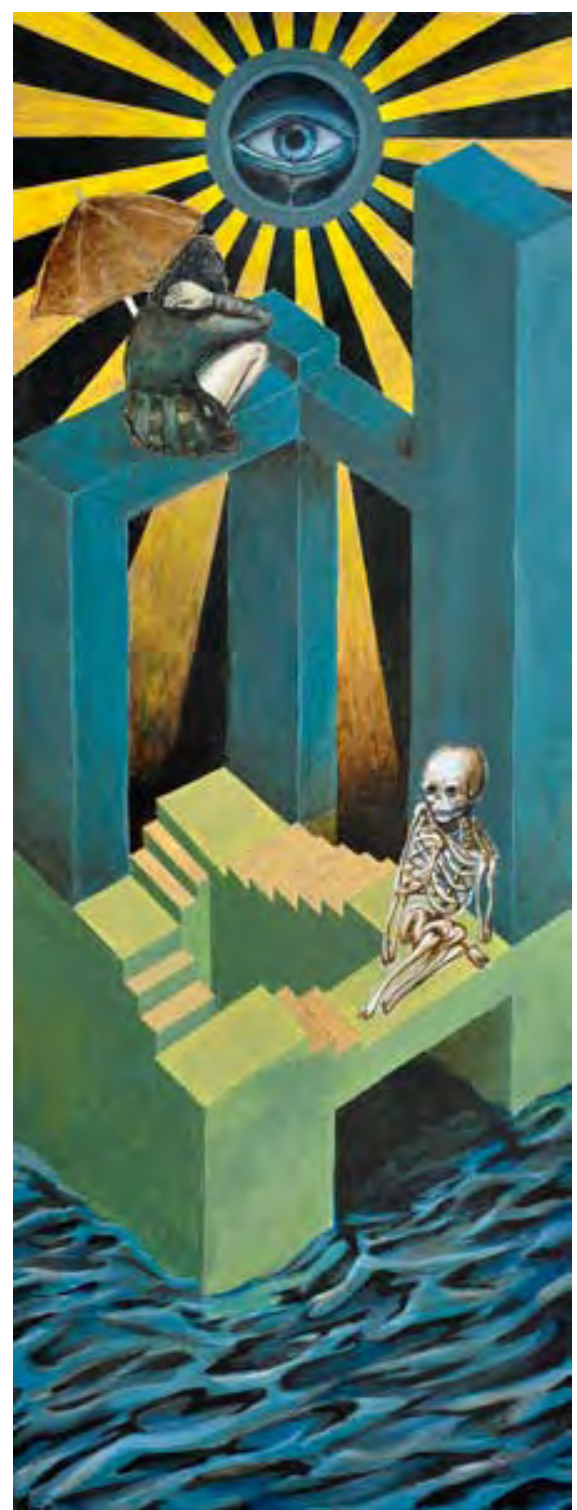

An Impossible Romance, 2008, Acrylic on plywood, $94 \times 35 \mathrm{~cm}$

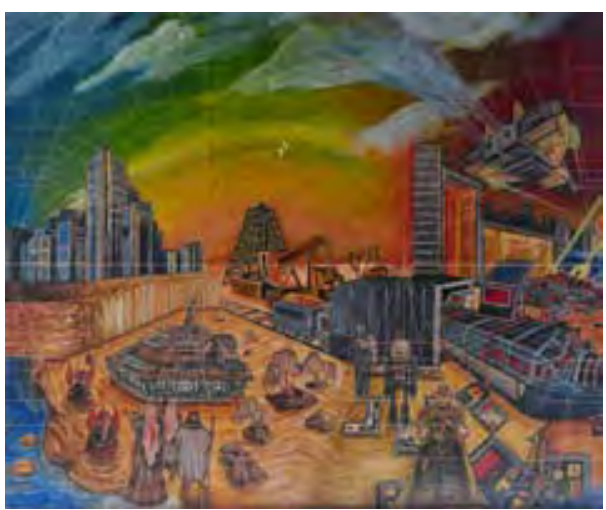

2. Our minds are capable of understanding spatial concepts beyond the reach of human senses.

Humans are built to perceive 3 dimensions. Whether it's because of natural selection or of intelligent design, we can't experience 4 or more, even if they are really there. But I believe the human mind is on the cusp of understanding that which we cannot directly experience. Of course there's a danger of hitting a dead end if we try to do so. Yet is the danger of finding nothing reason enough to stop our curiosity?

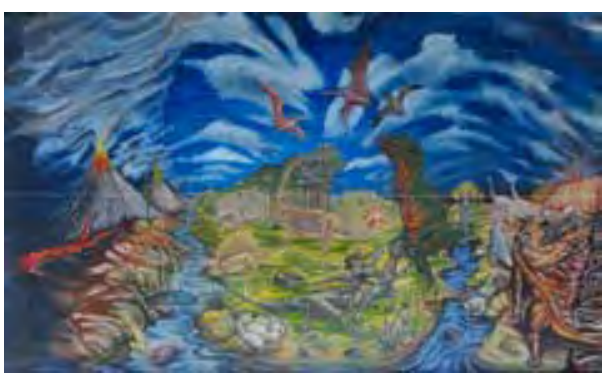




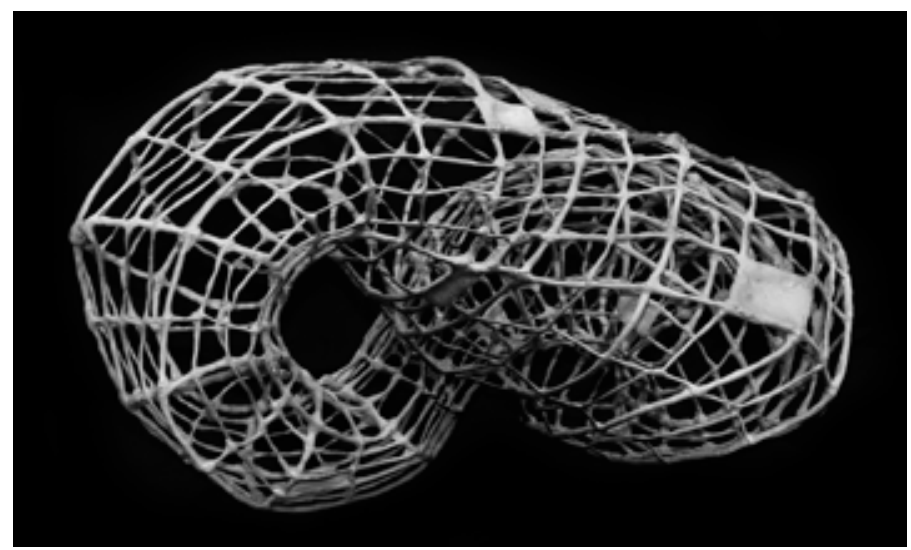

Klein Bottle 1, 2010 Epoxy on rubbercoated copper wire $33 \times 55 \times 35 \mathrm{~cm}$

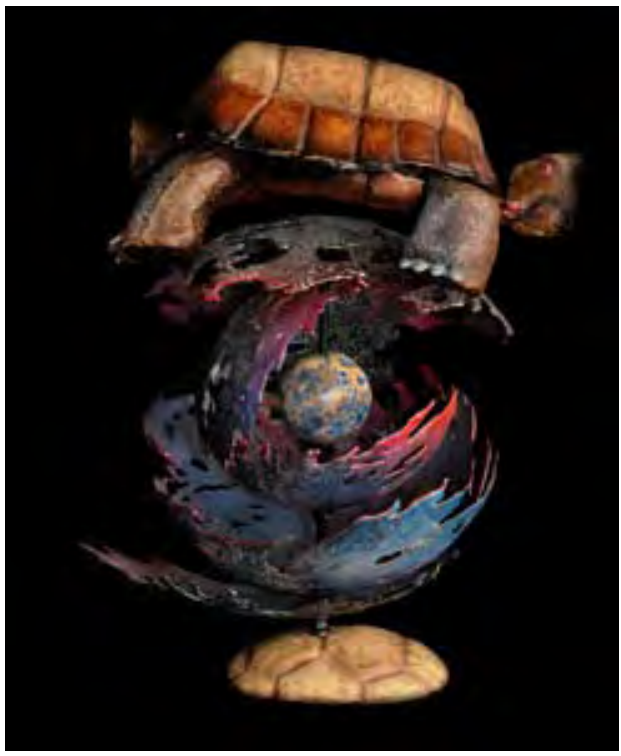

Carrying the World Whilst Being on It, 2010, Acrylic on epoxy, paper and metal $41 \times 41 \times 64 \mathrm{~cm}$

Mobius Strip, 2009, Acrylic on canvas, $121.92 \times 60.96 \mathrm{~cm}$

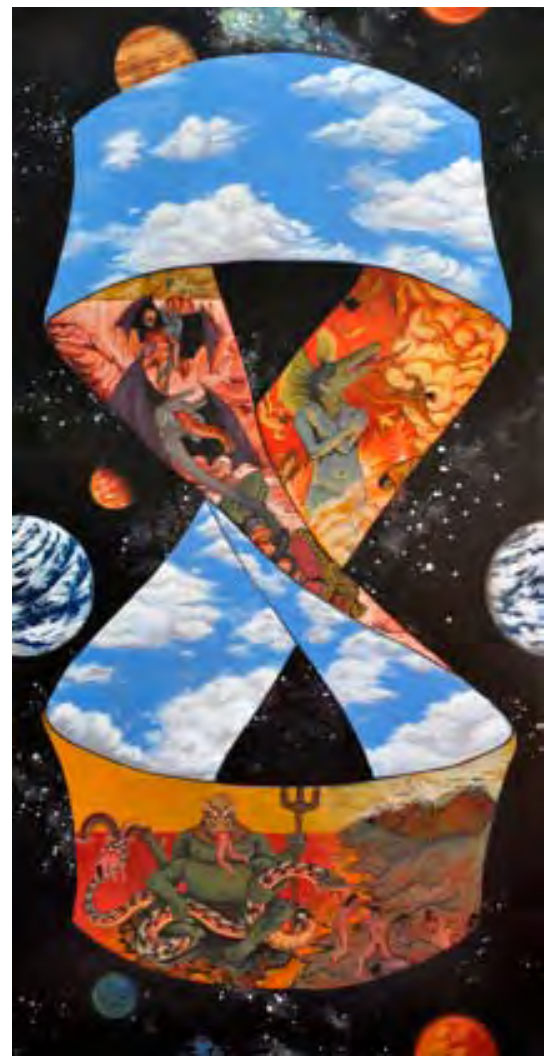




\section{Bottling up the Universe}

To the curious I say, rest assured, for there are tiny fragments of sense in dabbling about hidden dimensions. Through analogy and illustration we find visual tools to help us create our own personal understanding of spacehopefully one that may include the concept of hidden dimensions.

\section{The Mobius Strip}

Get a strip of an infinitely thin piece of paper. Twist once, then tape the two ends together with cosmic glue. What you get is a geometric 2-D shape bended in a 3-D space. Or we can say it's a 3-D shape made up of a single side and no corners. What you get is a mobius strip. It's a perfect model of how dimensions can be hidden.

Imagine what it would be like to be a 2-D inhabitant of a mobius strip universe. If you travel in one direction long enough, you'll find yourself back at your starting point.

\section{Misner Space}

One end is the beginning of the opposite end. It's a boundless universe. It's much like what you'd get if you unfold a mobius strip-only this time, the whole surface is connected.

\section{The Klein Bottle}

Think of it as a mobius strip with zero edges. Now, can you imagine a Klein bottle that doesn't intersect itself? I bet you can but you can never draw it.

\section{The Mobius Fractal: An Optical Joke}

If a Mobius strip is an analog for hidden dimensions, then making a fractal (geometric shape whose parts are a smaller version of the whole) out of it allows dimensions to be hidden infinite times. 
A closer look at the Mobius Fractal reveals that it is in fact, no more than a plain old garden variety Mobius strip fallaciously wrapped in false dimensions. The whole shape is still made of one surface.

\section{The Tesseract: A Four Dimensional Cube}

0-D points make 1-D lines make 2-D squares make 3-D cubes make 4-D tesseracts. Imagine a cube whose every face is a cube.

\section{Probabilities and Time}

What would happen if we throw in the concept of time in our soup of ideas? For one thing, it would give us another tool to describe or visualize dimensions with.

Plot out the history of the universe as we know it. Illustrate the different twists and turns it could have gone through. You'll end up with a tree of probabilities that behaves like a mobius fractal.

When you begin to think of dimensions with regards to this, you may begin to understand them extra spaces. A useful technique to imagine extra spaces is to imagine the presence of a different universe, one you cannot see or go to unless there is a portal or wormhole or break-in-the-spacetime-continuum.

Suppose the cogs of the universe are built this way, then our concept of time would need to be updated. The presence of extra hidden spaces indicates a possibility of creating infinite worlds. By infinite, I mean that there is room in reality for every single possible universe that could ever exist. Imagine a parallel universe where the big bang never happened. Imagine a universe where the atomic weight of copper is different from ours. Imagine a solar system with no earth, an earth where life never existed, or a world where birds evolve over apes. Imagine there is a parallel world out there that is exactly the same as the one you're in, except that you're wearing a different outfit. Imagine there is a parallel world out there that is exactly the same as the one you're in, except that it's one second later. This way, time begins to become less like a chronological line and more of moving from one probable state of matter to another. 
ASIAN PERSPECTIVES in the Arts and Humanities

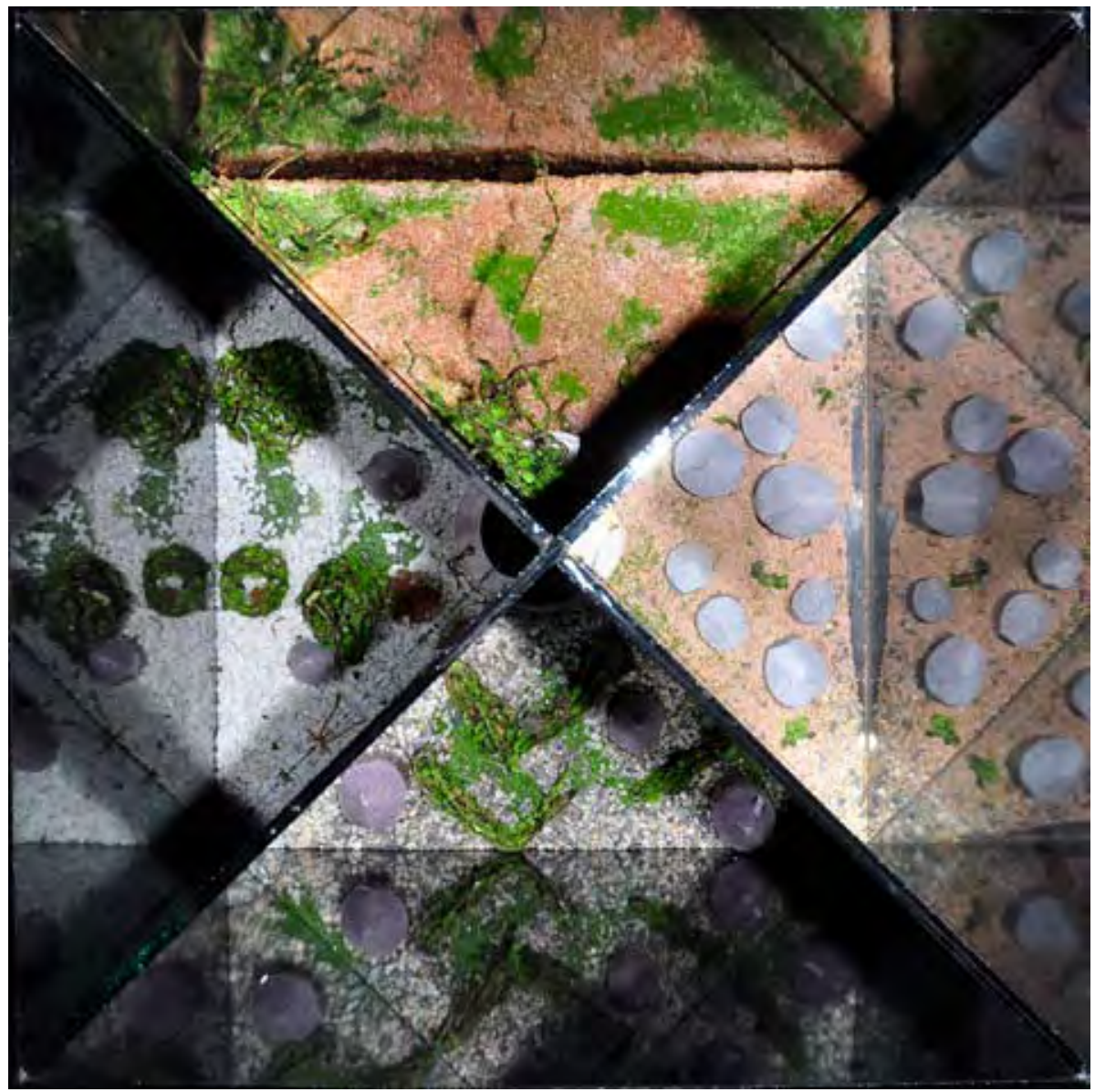

The Spacetime Tesseract, 2010, Glass, mirrors, gravel, readymade miniatures and digital print on paper, $38 \times 38 \times 38 \mathrm{~cm}$
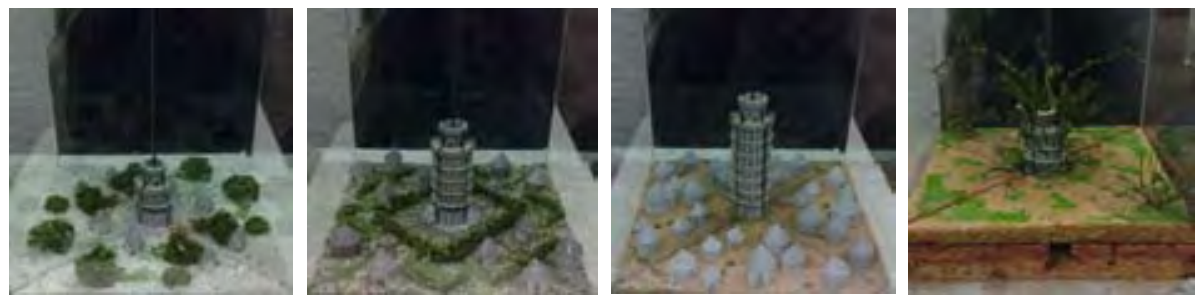